\title{
Human induced pluripotent stem cell-derived endothelial cells for modelling of endothelial dysfunction
}

\author{
Bing-li Liu ${ }^{1}$ Jian-hua $\mathrm{Ma}^{1 *}$ and Lei Ye ${ }^{2 *}$ \\ ${ }^{1}$ Department of Endocrinology, Nanjing First Hospital, Nanjing Medical University, Nanjing, China \\ ${ }^{2}$ National Heart Research Institute Singapore, National Heart Centre Singapore, Singapore, Singapore
}

\begin{abstract}
Human induced pluripotent stem cells (hiPSCs) cells are a type of cells derived from human somatic cells that are reprogrammed to an embryonic stem cell (ESC)-like pluripotent state. hiPSCs are being explored as disease modelling and drug discovery for diabetes mellitus. Modelling endothelial dysfunction using endothelail cells (ECs) derived from diabetic hiPSCs has the potential for evaluating the therapeutic effect of drugs or chemicals on endothelial dysfunction and for understanding the cross-talk between ECs and inflammatory cells or cytokines which will benefit the development of new therapeutic strategies for treatment of cardiovascular diseases.
\end{abstract}

\section{Editorial}

Human induced pluripotent stem cells (hiPSCs), a type of cells derived from human somatic cells, are reprogrammed to an embryonic stem cell (ESC)-like pluripotent state [1]. hiPSCs not only hold potential to provide immunologically compatible autologous cells for "personalized" cell transfer therapy, they also offer an opportunity for modelling human disease in cell culture dish to screen and identify drugs for treatment of inherited and acquired diseases. The differentiated cells from disease specific hiPSCs retain disease-related phenotypes which can be served as an in vitro model of pathogenesis. This provides an provides an innovative way to explore the molecular mechanisms of diseases [2].

Disease-specific hiPSC-ECs have been generated to study disease mechanisms or to screen drugs. Various disease-specific hiPSC lines have been generated and reported, including Moyamoya disease [3], fibrodysplasia ossificans progressive [4], the bone morphogenetic protein receptor type II (BMPR2) mutation [5], type 1 diabetes mellitus [6], type 2 diabetes mellitus [6], Huntington's disease [7], Kawasaki disease [8], atrial or ventricular septal defects, pulmonary valve stenosis, cardiomyopathy [9], calcified aortic valve disease [10], and hemophilia A [11]. They provide a unique opportunity to study underlying mechanisms of disease pathophysiology and establish a platform for high-throughput drug screening and toxicity testing.

Human iPSCs have been successfully differentiated into vascular cells, including endothelial cells (iECs) $[12,13]$ and smooth muscle cells (iSMCs) [14]. Earlier iEC differentiation protocol involved co-culture of hiPSCs with OP9 cells [15]. More recently, efficient iEC differentiation protocols, either 2-or 3-dimensional system, with defined factors have been developed $[12,13,16,17]$. iECs have typical EC characterizations: they express a panel of EC cell surface markers, such as CD31, CD144 and vWF-VIII, are able to uptake low-density lipoprotein and form tubular structure in vitro $[12,13]$. A large number of iECs can be obtained from hiPSCs and be used for treatment of peripheral artery disease, acute and chronic heart failure, stroke and certain forms of retinopathy associated with endothelial dysfunction to promote the repair of injured tissue.

The vascular ECs play an important role in cardiovascular homeostasis through paracrine factors that regulate vascular tone, fibrinolysis, cell adhesion, and blood flow $[18,19]$. It is known that endothelial dysfunction is a risk factor for cardiovascular disease (CVD). The iECs differentiated from iPSCs, which were reprogrammed from fibroblasts of mouse with diet-induced obesity, exhibited signs of endothelial dysfunction and had poor treatment efficacy following transplantation in a hind limb ischaemia model [20]. iPSCs from $\mathrm{db} /$ $\mathrm{db}$ mouse had impaired differentiation into CD34/Tie2 expressing endothelial progenitor-like cells and reduced angiogenic potential [21].

Modelling endothelial dysfunction using human diabetic iECs can be used to evaluate the therapeutic effect of drugs or chemicals on endothelial function and to understand the cross-talk between ECs and inflammatory cells or cytokines which will benefit the development of new therapeutic strategies for treatment of cardiovascular diseases.

Although much progress has been achieved recently, challenges remain in variability of generating different subtypes of iECs, stability of iEC expansion in vitro, and improving iEC function for treatment of ischemic disease, especially iECs differentiated from disease-specific hiPSCs [22].

${ }^{*}$ Correspondence to: $\mathrm{Ma} \mathrm{JH}$, Department of Endocrinology Nanjing Medical University Affiliated Nanjing Hospital 210006, Nanjing China, Tel: 8613905187504; Fax: 86-25-52887000; E-mail: majianhua@china.com

Ye L, MD, PhD, National Heart Research Institute Singapore, National Heart Centre Singapore, Singapore, Email: yeleislp@yahoo.com

Key words: induced pluripotent stem cells, endothelial dysfunction, disease modelling

Received: July 04, 2019; Accepted: July 10, 2019; Published: July 13, 2019 


\section{Declaration of interest}

The authors declare that there is no conflict of interest.

\section{Acknowledgement}

This study was supported by the National Natural Science Foundation of China (NO. 81870563).

\section{References}

1. Takahashi K, Tanabe K, Ohnuki M, Narita M, Ichisaka T, et al. (2007). Induction of pluripotent stem cells from adult human fibroblasts by defined factors. Cell 131: 861872 .

2. Kawser Hossain M, Abdal Dayem A, Han J, Kumar Saha S, Yang GM, et al. (2016) Recent Advances in Disease Modeling and Drug Discovery for Diabetes Mellitus Using Induced Pluripotent Stem Cells. Int J Mol Sci 17: 256.

3. Hamauchi S, Shichinohe H, Uchino H, Yamaguchi S, Nakayama N, et al. (2016) Cellular Functions and Gene and Protein Expression Profiles in Endothelial Cells Derived from Moyamoya Disease-Specific iPS Cells. PloS one 11: e0163561.

4. Barruet E, Morales BM, Lwin W, White MP, Theodoris CV, et al. (2016) The ACVR1 R206H mutation found in fibrodysplasia ossificans progressiva increases human induced pluripotent stem cell-derived endothelial cell formation and collagen production through BMP-mediated SMAD1/5/8 signaling. Stem Cell Res Ther 7: 115.

5. Gu M, Shao NY, Sa S, Li D, Termglinchan V, et al. (2017) Patient-Specific iPSC-Derived Endothelial Cells Uncover Pathways that Protect against Pulmonary Hypertension in BMPR2 Mutation Carriers. Cell stem cell 20: 490-504.

6. Teo AK, Gupta MK, Doria A, Kulkarni RN (2015) Dissecting diabetes/metabolic disease mechanisms using pluripotent stem cells and genome editing tools. Mol Metab 4: 593-604.

7. Lim RG, Quan C, Reyes-Ortiz AM, Lutz SE, Kedaigle AJ, et al. (2017) Huntington's Disease iPSC-Derived Brain Microvascular Endothelial Cells Reveal WNT-Mediated Angiogenic and Blood-Brain Barrier Deficits. Cell Rep 19:1365-1377.

8. Ikeda K, Mizoro Y, Ameku T, Nomiya Y, Mae SI, et al. (2016) Transcriptional Analysis of Intravenous Immunoglobulin Resistance in Kawasaki Disease Using an Induced Pluripotent Stem Cell Disease Model. Circulation journal: official journal of the Japanese Circulation Society 81: 110-118.

9. Ang YS, Rivas RN, Ribeiro AJS, Srivas R, Rivera J, et al. (2016) Disease Model of GATA4 Mutation Reveals Transcription Factor Cooperativity in Human Cardiogenesis. Cell 167: 1734-1749.
10. Theodoris CV, Li M, White MP, Liu L, He D, et al. (2015) Human disease modeling reveals integrated transcriptional and epigenetic mechanisms of NOTCH1 haploinsufficiency. Cell 160: 1072-1086.

11. Wu Y, Hu Z, Li Z, Pang J, Feng M, et al. (2016) In situ genetic correction of F8 intron 22 inversion in hemophilia A patient-specific iPSCs. Sci Rep 6: 18865.

12. Su L, Kong X, Lim S, Loo S, Tan S, et al. (2018) The prostaglandin H2 analog U-46619 improves the differentiation efficiency of human induced pluripotent stem cells into endothelial cells by activating both p38MAPK and ERK1/2 signaling pathways. Stem Cell Res Ther 9: 313.

13. Zhang S, Dutton JR, Su L, Zhang J and Ye L (2014) The influence of a spatiotemporal $3 \mathrm{D}$ environment on endothelial cell differentiation of human induced pluripotent stem cells. Biomaterials 35: 3786-3793

14. Maguire EM, Xiao Q, Xu Q (2017) Differentiation and Application of Induced Pluripotent Stem Cell-Derived Vascular Smooth Muscle Cells. Arterioscler Thromb Vasc Biol 37: 2026-2037.

15. Choi KD, Yu J, Smuga-Otto K, Salvagiotto G, Rehrauer W, et al. (2009) Hematopoietic and endothelial differentiation of human induced pluripotent stem cells. Stem Cells 27: 559-567

16. Lee SJ, Sohn YD, Andukuri A, Kim S, Byun J, et al. (2017) Enhanced Therapeutic and Long-Term Dynamic Vascularization Effects of Human Pluripotent Stem Cell-Derived Endothelial Cells Encapsulated in a Nanomatrix Gel. Circulation 136: 1939-1954.

17. Liu X, Qi J, Xu X, Zeisberg M, Guan K, et al. (2016) Differentiation of functional endothelial cells from human induced pluripotent stem cells: A novel, highly efficient and cost-effective method. Differentiation: research in biological diversity 92: 225236

18. Messner B and Bernhard D (2014) Smoking and cardiovascular disease: mechanisms of endothelial dysfunction and early atherogenesis. Arterioscler Thromb Vasc Biol 34: 509-515.

19. Lee WH, Ong SG, Zhou Y, Tian L, Bae HR, et al. (2019) Modeling Cardiovascular Risks of E-Cigarettes with Human-Induced Pluripotent Stem Cell-Derived Endothelial Cells. Journal of the American College of Cardiology 73: 2722-2737.

20. Gu M, Mordwinkin NM, Kooreman NG, Lee J, Wu H, et al. (2015) Pravastatin reverses obesity-induced dysfunction of induced pluripotent stem cell-derived endothelial cells via a nitric oxide-dependent mechanism. Eur Heart J 36: 806-816.

21. Stepniewski J, Kachamakova-Trojanowska N, Ogrocki D, Szopa M, Matlok M, et al. (2015) Induced pluripotent stem cells as a model for diabetes investigation. Sci Rep 5: 8597.

22. Carcamo-Orive I, Huang NF, Quertermous T and Knowles JW (2017) Induced Pluripotent Stem Cell-Derived Endothelial Cells in Insulin Resistance and Metabolic Syndrome. Arterioscler Thromb Vasc Biol 37: 2038-2042.

Copyright: (C2019 Liu B. This is an open-access article distributed under the terms of the Creative Commons Attribution License, which permits unrestricted use, distribution, and reproduction in any medium, provided the original author and source are credited. 